\title{
LA IMAGEN PROFESIONAL DEL PROFESORADO EN FORMACIÓN CONTINUA
}

\section{TEACHER'S PROFESSIONAL IMAGE IN IN-SERVICE TRAINING}

\author{
Zoe Martínez de la Hidalga \\ Lourdes Villardón-Gallego \\ Universidad de Deusto
}

Fecha de recepción: 12 de junio de 2013

Fecha de aceptación: 29 de junio de 2014

Fecha de publicación: 30 de junio de 2014

\section{RESUMEN}

La importancia del profesor como educador y formador de ciudadanos proactivos, competentes, flexibles y comprometidos es innegable. Por ello, es importante que los profesores sean competentes para cumplir adecuadamente con sus funciones.

Las creencias del profesor sobre la profesión docente, la enseñanza, la organización de clase, etc. influyen en su actuación docente. En consecuencia, es importante identificar dichas creencias como elemento de partida en la formación del profesorado.

Esta investigación tiene como objetivo conocer la imagen del profesor y la percepción de la enseñanza del profesorado en activo. Con este fin se ha encuestado a 17 profesores de Educación Infantil y Primaria en ejercicio, participantes en distintas actividades de formación continua organizadas y desarrolladas por la Universidad de Deusto durante el segundo semestre del curso 2012-2013.

Los profesores, a través de un cuestionario on line de preguntas abiertas, han explicado, su concepción de la profesión docente, han identificado las características de un buen profesor y las dificultades encontradas durante el desempeño profesional.

El análisis de contenido de las respuestas arroja información relevante, con implicaciones interesantes para la formación del profesorado.

Los resultados indican que los participantes son conscientes del perfil profesional en cuanto a funciones y competencias e identifican aspectos que dificultan y favorecen su desempeño. La experiencia profesional favorece una imagen más realista de la profesión.

Palabras clave: identidad profesional, formación de profesorado, metáfora, investigación cualitativa, perfil docente

\section{ABSTRACT}

The significance of a teacher as an educator and trainer of proactive, capable, flexible and committed citizens is undeniable. Thus, it is important that teachers be proficient to adequately fulfill their duties.

Teachers' beliefs about the teaching profession, training, class organization, etc. influence in their teaching behaviour. Therefore, it is important to identify these beliefs as a key element in the teachers' training.

The aim of this research is to determine the image of the teacher and the teaching perception in active teachers. The study involved a group of 17 Pre-primary and Primary 
La imagen profesional del profesorado en formación continua.

Education teachers, engaged in several training activities organized and carried out by the University of Deusto, during the second semester of the 2012-2013 academic years. Teachers, through an online questionnaire with open questions, have explained, among other things, their conception of teaching profession, the characteristics of being a good teacher as well as the difficulties they found during the professional performance.

The content analyses of the answers disclose relevant information, with interesting implications for teacher training.

Outcomes indicate that participants are aware of the professional profile in terms of roles and competences and identify issues that complicate and promote their teaching. Work experience encourages a more realistic image of the profession.

Key words: professional identity, teacher training, metaphor, qualitative research, teacher's profile.

\section{INTRODUCCIÓN}

La identidad profesional del profesor "se refiere a cómo los docentes viven subjetivamente su trabajo" (Marcelo y Vaillant, 201 1: 35). La identidad está formada por las ideas y concepciones subjetivas del profesor sobre su quehacer; las dificultades que encuentra en su tarea, las funciones y competencias que le son propias, las características que desde su punto de vista tiene que tener un buen docente, su concepto sobre el ser profesor. Por tanto, es un ejercicio de definición sobre uno mismo: qué soy, cómo soy, cómo quiero ser, cómo me ven, qué características tengo, que sé hacer, qué tengo que hacer. Todo este ejercicio de reflexión personal, de conocimiento de uno mismo (Lauriana y Kukkonen, 2005) irá conformando la identidad profesional del docente como "la síntesis de un proceso de identización o consideración de elementos de diferenciación de naturaleza biográfica o personal" (Galaz, 2011:91).

La identidad profesional es una parte principal del desarrollo profesional. Posibilita la construcción de las propias ideas sobre cómo ser, actuar, cómo considerar su desempeño y su papel en la sociedad (Sachs, 2005).

Cattonar (citado por Bolívar, 2006) dice que la imagen profesional es un modo de verse a sí mismo teniendo en cuenta las características que encontramos en uno mismo y en otros siendo un proceso biográfico continuo y un proceso relacional.

Para Tardiff (2004), la imagen profesional es un conjunto de saberes teóricos y experienciales y que se construye lentamente porque tiene en cuenta la socialización en la profesión y en su experiencia profesional. 
Para ser un buen profesor es necesaria la construcción de la identidad profesional. En palabras de Palmer (1998: 2) "si no me conozco a mí mismo, no puedo saber quiénes son mis alumnos... no puedo conocer mi materia...".

El profesor tiene que tener conocimientos y competencias propias de su desempeño profesional pero su identidad profesional tendrá un gran peso en el proceso de convertirse en un profesor como afirman Sutherland y Markauskaite (2012).

La identidad profesional del profesor no sólo está construida por la imagen profesional, individual de cada docente sino por las ideas colectivas de diferentes grupos de profesionales que trabajan juntos. Pero además, las identidades personales de cada profesor son un influjo claro en los profesionales:

"El modo en que los maestros forman sus identidades profesionales está influido tanto por lo que sienten de sí mismos como por lo que sienten de sus alumnos. Su identidad profesional les ayuda a situarse en relación con sus alumnos y a hacer ajustes adecuados y eficaces en su práctica y sus creencias sobre los estudiantes y su compromiso con ellos" (James-Wilson, 2001, citado por Day, 2006: 70).

En la identidad profesional del profesor tiene una influencia fundamental la emoción y el concepto de uno mismo. La emoción puede modificar la imagen profesional del profesor por lo que Timostsuk y Ugaste (2012) afirman que es importante trabajar la educación emocional en la formación del profesorado.

Otro aspecto interesante a tener en cuenta en la construcción de la identidad profesional del profesor es su imagen social. La identidad profesional del profesor se encuentra en peligro. Hace bastantes años, el ser profesor, educador, era considerado como una profesión respetable, con alto prestigio social. Esta situación ha cambiado considerablemente (Marcelo y Vaillant, 2011: 38). En nuestros días, la imagen social que muchos manifiestan sobre nuestra profesión es bastante negativa, todos pueden y saben hablar de educación y, además, muchos de los profesionales de la educación también lo creen. Bolívar (2006: 13) dice que "las mutaciones de las últimas décadas generan sobre la situación profesional de los profesores ambigüedades y contradicciones".

La identidad profesional del profesor se va forjando a lo largo de toda la carrera del docente $o$ incluso antes. Posiblemente se inicia 
La imagen profesional del profesorado en formación continua.

algún día mientras realiza sus propios estudios en las etapas de Educación Infantil, Primaria y/o Secundaria. Posteriormente, se consolida en su formación inicial como docente. En el caso de los maestros se irá fortaleciendo en sus estudios de Magisterio en Educación Infantil o Primaria. En el caso de los profesores de Secundaria irá perfilándose durante sus estudios específicos de Historia, Literatura, Lengua, Idiomas, Matemáticas, Física,... Posteriormente, accederá al Máster de Secundarial y será en estos estudios donde su acercamiento a la realidad docente será mayor. En esta formación inicial no queda consolidada la identidad profesional del docente sino que continuará durante su ejercicio profesional como educador. Como dicen Beijard, Meijer y Verloop (2004) y Galaz (2011) la identidad no es algo que uno tiene sino que se desarrolla a lo largo de la vida.

La identidad profesional del profesor tiene que ser uno de los elementos clave en cualquier proceso de formación docente (Bolívar, 2006 citado por Tiana, 2011) inicial o continuo. Los programas de formación inicial del profesorado deben desarrollar la identidad profesional por lo que habrá que utilizar metodologías adecuadas para ello como la reflexión sobre la acción (Walkington, 2005) y los foros de discusión entre estudiantes y profesores en ejercicio (Sutherland y Markauskaite, 2012). Esto permitirá avanzar en el desarrollo y fortalecimiento de su propia identidad.

La formación del profesor debe tener un componente eminentemente práctico por la "necesidad de construir una identidad profesional, lo que supone transformar a los estudiantes en docentes" (Tiana, 2011: 19).

Al comenzar el ejercicio profesional, la imagen del profesor cambia $y$, por tanto, es un momento importante en el desarrollo de la identidad profesional.

\section{METODOLOGÍA}

En este apartado se describen los diferentes elementos del diseño llevado a cabo en esta investigación con el fin de conseguir el objetivo planteado.

La población está constituida por el profesorado participante en los cursos de Formación Continua organizados por la Facultad de

\footnotetext{
1 Máster Universitario en Formación del Profesorado de Educación Secundaria Obligatoria, Bachillerato, Formación Profesional y Enseñanza de Idiomas
} 
Psicología y Educación de la Universidad de Deusto durante el segundo semestre del curso 2012-13.

Los participantes voluntarios en el estudio han sido un total de 17 profesores en activo de Educación Infantil y Primaria. La muestra proviene de tres actividades formativas llevadas a cabo en el periodo indicado. En la actividad formativa A participaron 19 profesores, de los cuales respondieron, 4. En la actividad B había 20 participantes y únicamente respondió uno y en la actividad $C$ se inscribieron 19, de los que respondieron 12 profesores.

La distribución de la muestra en base a las características sociodemográficas aparece en la Tabla 1.

\begin{tabular}{|c|c|c|}
\hline Variable & $\mathbf{N}$ & $\%$ \\
\hline \multicolumn{3}{|l|}{ Edad } \\
\hline De 25 a 35 años & 8 & 47 \\
\hline De 36 a 45 años & 7 & 41 \\
\hline De 46 a 50 años & 2 & 12 \\
\hline \multicolumn{3}{|l|}{ Sexo } \\
\hline Femenino & 16 & 94 \\
\hline Masculino & 1 & 6 \\
\hline \multicolumn{3}{|l|}{ Red } \\
\hline Concertada & 5 & 29 \\
\hline Pública & 12 & 71 \\
\hline \multicolumn{3}{|l|}{ Experiencia (años) } \\
\hline De 2 a 5 & 7 & 41 \\
\hline De 6 a 10 & 5 & 29 \\
\hline De 11 a 15 & 4 & 24 \\
\hline De 16 a 20 & 1 & 6 \\
\hline \multicolumn{3}{|l|}{ Nivel Educativo } \\
\hline Educación Infantil & 7 & 41 \\
\hline Educación Primaria & 10 & 59 \\
\hline
\end{tabular}

Tabla 1. Características sociodemográficas de los participantes

Como se puede observar, cerca del $90 \%$ son menores de 45 años, la inmensa mayoría son mujeres y pertenecientes a centros de la red pública. Un $40 \%$ tiene menos de 5 años de experiencia y solo un $6 \%$ tiene más de 16. Casi un $60 \%$ son maestros de Educación Primaria, y el resto de Educación Infantil.

El instrumento utilizado para la recogida de información ha sido un cuestionario on line con dos partes diferenciadas. La primera, con preguntas cerradas, referida a las variables de identificación y de recorrido académico y profesional; y la segunda, con preguntas abiertas, relacionadas con aspectos vinculados a la concepción de la profesión docente, tales como las funciones, competencias y dificultades en el desempeño. El cuestionario se elaboró a partir de los 
La imagen profesional del profesorado en formación continua.

objetivos del estudio de forma consensuada por cuatro miembros del equipo de esta investigación, los cuatro profesores universitarios del área de Educación y expertos en metodología de la investigación. Antes de la aplicación definitiva del cuestionario a los participantes del estudio, se comprobó la comprensibilidad de las preguntas a través de una aplicación previa a un grupo de 10 estudiantes del Master de Educación Secundaria.

El cuestionario definitivo incluye preguntas referidas al perfil del profesor (¿Cuáles son las funciones del profesor? ¿Qué tiene que saber hacer?), al desempeño docente (Indique las principales dificultades que tiene que afrontar un/a profesor/a en su desempeño profesional; ¿Qué aspectos ayudan al profesor/a a desempeñar sus funciones?), a la imagen profesional (¿Qué es para usted ser profesor/a? Elija una metáfora que describa la figura del profesor/a. ¿Qué características tiene un/a buen/a profesor/a? ¿Qué imagen social tiene la profesión de profesor/a?) y a la evolución de la imagen del profesor (¿̇Cómo ha cambiado su imagen del profesor/a desde que comenzó a trabajar como docente hasta la actualidad?)

El procedimiento llevado a cabo para la aplicación de los cuestionarios ha seguido los siguientes pasos:

1. Información presencial sobre la investigación el último día de clase de la actividad formativa y petición de colaboración de forma voluntaria y anónima.

2. Envío por email a cada uno de los profesores y profesoras que libremente accedieron a formar parte de la investigación de la dirección web para responder.

La información recogida en los cuestionarios fue analizada a través del establecimiento de categorías de respuesta para cada pregunta abierta formulada siguiendo un procedimiento inductivo.

\section{RESULTADOS}

Se realizó un análisis del contenido de las respuestas estableciendo a posteriori las categorías de respuesta. Se presentan los resultados siguiendo las dimensiones del cuestionario. 


\subsection{Perfil del profesor}

Para conocer cómo configuran los encuestados el perfil del profesor, se les ha preguntado por las funciones y las competencias propias de la profesión.

En cuanto a las funciones del profesor, se diferencian las funciones "formativas y educativas" (C02). Las formativas se centran en "llevar a cabo un curriculum que está programado" (C02), "enseñar" tanto "conocimientos como destrezas" (C01, C03, C07, C10) y "guiar al alumno en el proceso de aprendizaje" (C11), "enseñar a aprender" (C14), y "hacer que los alumnos entiendan la materia impartida" (C13).

Las funciones educativas se explicitan a través de expresiones como "transmitir (C01, C17) e inculcar valores" (C03), "educar" (C07), "convivir y enseñar a convivir" (C07).

Para llevar a cabo estas funciones algunos participantes han señalado algunas tareas que debe realizar el docente:

- Acompañar (C05; C07), guiar, aconsejar (C06) y apoyar al alumno (C01).

- Transmitir conocimiento (C07).

- Orientar (C07; C17), ofrecer tutoría y ejemplo (C16).

- Establecer un clima de confianza y seguridad en el que los niños y niñas se sientan seguros y acogidos $(\mathrm{C} 10 ; \mathrm{C} 14)$ para fomentar la participación de los alumnos (C10) y el desarrollo emocional (C14), así como la autoestima (C01) y la integración (C08; C10).

- Enseñar habilidades para la vida (C10).

- Atender las necesidades (C17) e intereses (C14) de cada alumno.

- Planificar, preparar las clases y los materiales (C15).

- Motivar (C16).

Para la realización de estas tareas y el desempeño de estas funciones el docente "debe conocer las características individuales y grupales de los alumnos (C15; C!7), "sus intereses, motivaciones, zona de desarrollo próximo, estilos de aprendizaje" (C14). 
La imagen profesional del profesorado en formación continua.

Algún participante indica sentirse "sobrepasado" por la asunción de algunas funciones que cree que no le corresponden e indica que "hoy en día tenemos que actuar de padres y madres... en muchos casos los padres nos dejan esta labor -educativa- en nuestras manos como si fuera nuestra obligación y por la cual estamos contratados" (C02). "Actualmente creo que las funciones de los profesores no están muy delimitadas, deberíamos enseñar, procurar que los niños con nuestra ayuda consiguiesen sacar el máximo partido a su capacidad de aprender, pero la educación debería recaer en los padres." (C04).

Las respuestas de los participantes a la pregunta sobre las competencias del profesor, es decir, sobre qué tiene que saber hacer, se pueden agrupar siguiendo los elementos de la competencia (conocimientos, habilidades y actitudes). En el primer grupo, estarían respuestas como las siguientes: "se le exige que sepa un poco de todo, desde conocer distintos idiomas, conocer las nuevas tecnologías, también tiene que saber cantar, bailar..." (C12); "Conocer las características psicológicas del que tiene enfrente" (C03); "Debe conocer la materia a enseñar, adquirir conocimientos teóricos sobre el aprendizaje y aprendizaje de las ciencias" (C15). Con respecto a las habilidades se mencionan, por ejemplo:

- "enseñar de manera que llegue a sus alumnos, que les empape" (CO1).

- "transmitir la información de diferentes modos para que llegue a todos los alumnos." (C03), "transmitir conocimientos" (C14), "ajustar la metodología en función de las demandas" (C03).

- "captar la atención de sus alumnos. No hacer las clases aburridas y monótonas" (C06); "mantener a sus alumnos motivados para aprender" (C05); motivar a su grupo creando un clima favorable para ello (C14); "captar la atención y de un modo entretenido... dar pautas de estudio..." (C14).

- "observar de forma continua para detectar posibles casos de dificultades de aprendizaje" (CO3).

- "trabajar habilidades sociales, trabajar la empatía" (C 10).

- "transmitir valores" (C01; C10).

- "preparar las actividades, dirigir la actividad de los alumnos, evaluar, utilizar la investigación e innovación" (C17). 
- Los encuestados incluyen, asimismo, respuestas referidas a las actitudes del profesor:

- "tiene que ser amable, comprensivo y empático" (C01).

- "respetar el ritmo individual de cada persona y apoyarlo durante todo el proceso educativo" (C09).

- "ser comprensivo, paciente, saber escuchar... y llegar afectivamente al máximo de alumnos" (C10).

- "gustarte tu trabajo y ser feliz realizándolo" (C10).

Algunos maestros y maestras son conscientes de la dificultad que entraña el desempeño de las funciones docentes, para lo que se requiere un alto nivel de competencia: "llevo poco tiempo en la enseñanza, pero el suficiente para darme cuenta que realmente se necesitan una gran variedad de habilidades para desempeñar este trabajo" (C13). Así, afirman que el profesor debe saber "lo que en el momento y la situación determinada sea necesario, sobre todo para el pleno desarrollo del alumno, pero también para el suyo propio" (C07), debe ser capaz de "tener en cuenta todo lo que le rodea para poder aplicarlo de una forma eficaz a sus funciones como docente" (C11).

En resumen, los participantes son conscientes de las dos funciones principales de los maestros: enseñar y educar. Para su desempeño indican que el profesor debe desarrollar competencias que incluyen la adquisición de conocimientos (por ejemplo, sobre didáctica, sobre las materias, sobre psicología...), habilidades (por ejemplo, comunicar, interesar, planificar actividades...) y actitudes (como empatía, comprensión, amabilidad, motivación...).

\subsection{Desempeño docente}

Para conocer la opinión de los participantes sobre el desempeño docente se les ha preguntado por las dificultades que encuentran y por los aspectos que ayudan a llevar a cabo sus funciones.

Los profesores señalaron dificultades relacionadas con los recursos, tales como el tiempo o la falta de personal de apoyo, así como el excesivo número de alumnos por aula:

- "La principal dificultad, en mi opinión, es la falta de tiempo. No llegamos donde nos gustaría por estar sometidas a una presión ejercida por el tiempo" (C02). "Falta de tiempo para poder trabajar con todo el grupo de clase" (C07). "Poco tiempo en el 
La imagen profesional del profesorado en formación continua.

colegio para elaborar actividades... Escaso tiempo para la coordinación entre paralelas" (C11).

- "Tiempos para la formación (mayoría fuera del horario laboral)" (C06).

- "Falta de recursos humanos... dificultades de aprendizaje del alumno y por no estar en el listado de NEE2 no puede recibir horas de apoyo..." (C06). "Cada vez menos recursos personales en situaciones en las que hace falta, lo que provoca sensación de soledad: arréglate como puedas" (C15). "Falta de especialistas en apoyo educativo" (C10). "La actual crisis que hace que cada vez haya más recortes" (C 14).

- "Número de alumnos por aula..." (C06; C11). "Grupos cada vez más numerosos, con más dificultades..." (C15).

- "Papeleo, papeleo y más papeleo que resta tiempo para dedicar a las niñas y niños" (C15).

Otro tipo de dificultades están relacionadas directamente con las características de los estudiantes, diversos y poco motivados:

- "... y perfiles de alumnos dispares" (C06). "Diferentes niveles de aprendizaje en el mismo aula" (C07). "Niños y niñas cada vez con problemáticas, más diversas" (C15).

- "El profesor de hoy en día se enfrenta a un tipo de alumnado cada vez más desinteresado, más sobreprotegido que representa actitudes de poco respeto hacia el mismo y hacia los demás" (C08). "Falta de motivación de los alumnos. Desgaste psicológico que implica tener que lidiar con el alumnado de hoy en día carente en muchas ocasiones de normas de comportamiento y convivencia" (C04). "En muchas ocasiones la falta de motivación inicial del alumno" (C 16). "La desmotivación de los niños" (C12).

Los profesores encuentran dificultades en las actitudes y comportamientos de otros implicados, como las familias y otros profesionales:

- "Falta de colaboración de las familias y poca implicación para solucionar los problemas de sus hijos" (C04). "El trato con algunos padres, que no quieren reconocer cómo son sus hijos y piensan

2 NEE (Necesidades Educativas Especiales) 
que eres su enemigo" (C05). "Falta de motivación de algunos padres para implicarse en la educación de sus hijos/as" (C07). "La poca participación de los padres en las actividades del colegio" (C11). "Se piensa que el profesor tiene que educar al niño. La base de la educación está en la familia y muchas veces nos encontramos que no podemos hacer mucho más si la familia o el entorno familiar no pone nada de su parte" (C13). "La familia, que en algunas situaciones se niega a colaborar con el centro" (C 14). "La permisividad de algunos padres" (C12). "Realizamos tareas que corresponden a las familias. Cada vez recibimos menos apoyo de las familias" (C10).

- "La mayor dificultad es el lidiar con tantos profesionales y llegar a un acuerdo de cómo enfocar la ayuda que se le va a dar al alumno" (C09). "Además de las dificultades que se encuentran en el alumnado y con la comunidad educativa, problemas con los padres" (C01).

Aspectos vinculados al reconocimiento social suponen una dificultad para el profesorado:

- "Sobre nuestra profesión todas las personas saben o entienden aunque luego en la realidad no tienen ni idea. Durante muchísimos años han sido los estudios de los "paracaidistas" con lo cual la profesionalidad se ha puesto muchas veces en tela de juicio" (C03). "La trivialización de la profesión -todo el mundo es maestro-, no es necesario tener formación alguna" (C06).

- "Margen de maniobra inexistente en decisiones curriculares básicas (ejemplo: las ACls son los padres los que las tienen que aprobar, dificultades de aprendizaje del alumno o alumna" (C06).

- "Las familias actuales han despojado al profesor del papel que tenía antaño y se le ha hecho perder autoridad. Se enfrenta a cambios sociales, familiares, diversidad de alumnado y ámbitos sociales...." (C08).

- "Cada vez se pone más en duda nuestra labor" (C 10). "La escasa valoración de los padres sobre nuestro trabajo" (C11); "El desprestigio al que se ven sometidos algunos profesionales por parte de la sociedad en general" (C12).

- "El apoyo por parte de todos los estamentos de la sociedad en este país nunca ha ocurrido, ni ocurre. Pues en muchos casos estamos ninguneados" (C03). 
La imagen profesional del profesorado en formación continua.

Entre los aspectos que ayudan a desempeñar la tarea de profesor, se señalan aquellos que se refieren a las características y condiciones propias del profesional: "el conocimiento que el profesor tiene, la cercanía para con sus alumnos..." (C02); "es imprescindible la motivación y las ganas de enseñar" (C08).

Uno de los aspectos mencionados con mayor frecuencia por los participantes como elemento facilitador de la tarea es el apoyo social de diferentes agentes relacionados con la función educativa:

- " "El apoyo de la dirección y el compañerismo" (C05). "El apoyo de la propia comunidad educativa" (C01).

- "La coordinación entre los docentes del colegio y la valoración y participación de los padres" (C 11). "Buena coordinación y trabajo en equipo" (C13). "La colaboración entre el profesorado" (C 14).

- "Que haya buen ambiente de trabajo" (C13); "estar a gusto en el centro educativo, sentirse arropado, uno más ... Tener un profesor paralelo con la que intercambiar impresiones, dudas ..." (C15).

- "Unas familias agradables en el trato" (C02). "Estrecha relación entre profesorado y familia y estar en sintonía" (C04).

- "Que los alumnos tengan unas normas mínimas de comportamiento y convivencia. Que estén motivados y tengan ganas de aprender" (C04).

Por supuesto, se consideran útiles diferentes tipos de recursos, tanto personales como materiales, ya que ayudan a desempeñar las tareas del profesor:

- "Personal de apoyo (PT3, PRL4 ...). Los recursos digitales" (C02). "Poder disponer de la ayuda de otro profesor, PT o Especialista de Apoyo Educativo en su clase" (C07).

- "Un buen funcionamiento y gestión desde el centro ayudará al desempeño de su labor" (C08). "Equipo directivo que deje bien claro cuáles son las funciones del profesorado" (C13).

- "La formación" (C02) y "El reciclaje" (C05).

3 PT (Pedagogo Terapéutico)

4 PRL (Profesor de Refuerzo Lingüístico) 
- "La tecnología y todos los avances ayudan mucho a preparar, transmitir, impartir..." (C08). "Los libros, la tecnología" (C01). "Los medios didácticos, que facilitan información y ofrecen interacciones facilitadoras de aprendizajes a los estudiantes" (C17).

- "Menos ratio en las aulas para trabajar mejor. Más horas libres en el colegio para desarrollar actividades" (C11).

- "Tener un grupo no muy numeroso..." (C15). "No tener un gran número de alumnos en clase. Que el nivel de clase sea equitativo" (C07).

En definitiva, según los participantes, el desempeño docente no está exento de dificultades, relacionadas, por un lado, por la falta de tiempo y de recursos, por las características de los alumnos, desmotivados y diversos, y por la falta de reconocimiento, apoyo social y "autoridad". Entre los elementos que ayudan a paliar estas dificultades se citan la motivación y capacitación del propio profesor, la coordinación entre profesores, el clima y buen ambiente de trabajo, el apoyo de las familias, los recursos materiales y personales, así como la formación.

\subsection{Imagen profesional}

El acercamiento a la imagen del profesor que tienen los maestros y maestras participantes se ha realizado a través de la definición del profesor, la elección y justificación de una metáfora que represente la profesión, la enumeración de las características de un buen profesor y el prestigio social percibido de la profesión.

A los participantes, se les pidió definir la profesión de profesor a través de la pregunta "qué es para usted ser profesor". Algunas de las respuestas dadas hacen referencia a la vocación que implica, debido al compromiso que supone: "Un tema vocacional. Algo que te tiene que gustar" (C02); "para mi ser maestra es una de las más nobles vocaciones" (C17).

Otros participantes describen ser profesor desde la satisfacción que les produce su desempeño: "es una experiencia muy enriquecedora dentro de la cual vas viendo los avances de los niños" (C12); "a mí personalmente mi trabajo me encanta y me resulta gratificante trabajar con niño/as de estas edades" (C15). 
La imagen profesional del profesorado en formación continua.

Algunos realizan una descripción a partir de las funciones de maestro, insistiendo en la función de guía para el aprendizaje y el desarrollo del estudiante, además de en la función de enseñar:

- "Ayudar en la formación integral del alumno sentando las bases para favorecer su proceso de enseñanza aprendizaje" (C03); "contribuir en la educación de los niños/as, apoyar a las familias en las dificultades,..." (C10).

- "... es aquella persona que se encarga de la enseñanza y la educación de los niños/as" (C04); "instrumento que guía a sus alumnos/as en su aprendizaje" (C05); "guía para el alumno/a" (C13).

- "... es una profesión que implica enseñar y transmitir una serie de conocimientos, experiencias, valores, trabajar y desarrollar las capacidades del alumno/a, su autonomía, su capacidad de decisión" (C08).

- "... ser profesor es algo más que impartir una materia a unos determinados alumnos; sobre todo cuando éstos cursan etapas iniciales de educación... significa acompañar al alumno en la tarea de ser formado en los distintos ámbitos que a lo largo de la vida va a tener que desempeñar" (Cl4).

Otros encuestados hacen referencia a la importancia de la profesión, y, por ello, a la necesidad de implicarse en el trabajo y de considerar a todos los estudiantes:

- "Por otra parte, mi papel es un factor importante en el desempeño de la sociedad,... en mis manos está el buen desarrollo de mis alumnos/as y la construcción de sus valores y actitudes" (C17).

- "Ser el responsable de favorecer el crecimiento personal, emocional y académico de los niños/as poniendo para ello todos los medios materiales y humanos disponibles en nuestras manos" (C15).

- "Enseñar significativamente conocimientos, valores.... a todos y cada uno de los alumnos/as" (C11).

- "Las mentes de mis alumnos es mi principal material, algo nada sencillo, y que requiere una labor enorme" (C17). 
A través de la metáfora se relacionan las ideas, palabras y el discurso analítico con diferentes imágenes (Sáez y García, 2011: 8). Algunas metáforas hacen referencia al profesor como fuente de conocimiento e inspiración, como un libro o un cuadro:

- "El profesor/a es como un libro abierto, del que todos sus alumnos/as pueden enriquecerse. Un libro porque enseña y muestra todo su contenido. Al acercarnos a un libro adquirimos su conocimiento y lo interiorizamos, al igual que los alumnos se acercan a nosotros e interiorizan lo que les enseñamos" (C02).

- "El profesor es como un libro o un cuadro que hay que mirar despacio para ver en su interior los detalles que en un primer vistazo se escapan, por ello hay una primera impresión visual (introducción al tema) y luego detalles menos perceptibles en una primer vistazo (aquellos detalles que el profesor nos va relatando y haciendo ver y entender" (C16).

En los participantes está muy presente la función del guía y orientación, a través de imágenes como pastor, entre otras:

- "El profesor es el guía que orienta a sus alumnos desde sus primeros años dándoles las herramientas necesarias para favorecer su proceso de enseñanza aprendizaje" (C04).

- "Un pastor que intenta cuidar y enseñar a su rebaño el buen camino" (C05): "un guía espiritual" (C06).

- "Un adivino. Tiene que saber lo que piensa cada niño para poder aplicarlo en su desempeño" (C12); "la profesora es como unas manos llenas de comprensión" (C11).

Algunas metáforas recogen la idea de que el profesor es un recurso, muy conscientes de que una de las metas educativas es el desarrollo de la autonomía del estudiante:

- "Como una cachaba. Los que se apoyan en ella pueden llegar a la meta, incluso a veces pueden llegar a prescindir de dicha cachaba... la cachaba es el símbolo más universal que representa la ayuda" (C09).

- "El profesor es la herramienta que necesita el alumno para alcanzar objetivos necesarios para desenvolverse en el medio" (C08). 
La imagen profesional del profesorado en formación continua.

Varios participantes han elegido una imagen que refleja su implicación y dedicación, aunque no siempre se logren los resultados esperados:

- "La de la hormiga y la cigarra. El profesor sería la hormiga, ya que poco a poco y con esfuerzo, conseguirá los objetivos establecidos con sus alumnos. Le costará más llegar hasta algunos, pero con otros su esfuerzo será pronto recompensado" (C07).

- "Un jardinero que cultiva, cuida, abona y riega sus flores para que un día florezcan" (C15). "El profesor es como un jardinero que cuida de sus plantas con mucho esmero e intenta que crezcan lo más bonitas y sanas posibles. Independientemente de la tierra en la que se han plantado. Realmente creo que intentamos hacer todo lo que podemos por los alumnos, pero no podemos intervenir en todas las situaciones -sobre todo familiares- por las que atraviesan algunos de ellos" (C14).

Algunas metáforas dejan al descubierto cierto descontento con el reconocimiento social de la profesión y con el desempeño en el día a día:

- "Un comodín. Sirve para todo y para nada. En caso de problemas somos el saco de los palos y en el caso de no haber problemas nunca o rara vez oirás una palabra de aliento" (C03).

- "Saco donde te pueden dar mil golpes" (C13).

- "El profesor es como una madre/padre. En muchas ocasiones la propia familia nos exige tomar decisiones que les competen a ellos mismos" (C10).

No obstante, alguno es consciente de la repercusión que tiene el profesor en la vida de los estudiantes, tanto en el presente como en el futuro:

- "Los inolvidables que marcan. Para mí el docente se convierte en un verdadero ejemplo y son guías de toda la vida y que aunque ya no se encuentren físicamente, sus obras y sus enseñanzas, jamás se olvidan. Siguen siendo el libro especial que permite seguir el camino correcto" (C17).

Cuando se pregunta a los maestros y maestras por las características de un buen profesor se mencionan las habilidades sociales: 
- Comunicación: "Buen comunicador... buen oyente e interpretador de lo que oye." (C15); "saber escuchar" (C09; C10; C05); "capacidad de comunicar" (C07).

- Empatía (C01; C03; C07; C13; C14; C15).

- Resolución de conflictos: "buen observador y mediador" (C14); "sabe mediar" (C05); "conoce a los alumnos" (C08).

Asimismo, consideran que el profesor tiene que estar motivado y preocupado por mejorar en su desempeño:

- "Estar abierta a la innovación y mejora continua" (C03; "...quien quiere mejorar y mejora, quien analiza para luego cambiar o seguir, quien se cree lo que está haciendo..." (C05); "con ganas de seguir aprendiendo y poniéndolo en práctica" (C13); "ganas de mejorar y de aprender" (C 14); "motivada, con ganas e interés por transmitir lo conocido y aprender lo nuevo para seguir transmitiendo. Ha de ser una persona que se adapta a los cambios y que se renueva con ellos" (C07).

- "ganas de enseñar" (CO1): "convierte su labor en un placer" (C16); "con voluntad, dedicación, ilusión" (C13).

En opinión de los participantes un buen profesor se caracteriza por su cercanía y preocupación por todos los alumnos que valora la diferencia:

- "Cercanía" (C01, C07; C09)C0) y comprensión (C04, C10; C16); "cariñosa" (C04); "cálido" (C05); "amigable" (C16); "llega afectivamente al máximo de alumnos" (C10); "da confianza al alumno para que ante cualquier problema acuda a él" (C12).

- "Aquel o aquella persona que se preocupa antes por la persona niño- que por el curriculum" (CO2); "Interesado en sus alumnos" (C16), "Dedica mucho tiempo a los alumnos y alumnas" (C14).

- "Responde a los intereses y expectativas de sus alumnos" (C03); "tiene que estar abierto a la diversidad y vivirla como un enriquecimiento tanto profesional como personal. Para ello debe escuchar a cada uno de los niñós/as del aula" (C11); "el que escucha al alumno e intenta ofrecerle unos recursos para que él pueda encontrar la solución a algunos problemas, para que pueda ir creciendo." (C12); "tiene en cuenta la diversidad y la respeta" (C09). 
La imagen profesional del profesorado en formación continua.

Algunos mencionan características personales del buen profesor:

- "Alegre, optimista, jovial, con buen sentido del humor" (C16); "Dinámico" (C15); "Activa" (C 13); "Creativa" (C10).

- "Paciencia" (C 16; C 17; C 10; C06; C04; C 14), "Tranquilo" (C 13).

- "Bondadoso, simpático" (C 16); "Amable" (C01).

- "Flexible (C09).

- "No se siente superior ni importante; no pretende saberlo todo" (C16).

Otros indican determinados estilos de desempeñar su trabajo:

- "No ser obstinado en llegar a conseguir todos los objetivos de clase" (C06).

- "Dinamizadora" (C10).

- "Favorece la autonomía y la toma de decisiones, hace ver que cada uno somos responsables de nuestras acciones tanto en lo bueno como en lo malo" (C14).

- "...y no tanto en hacer fichas. Es consciente de que muchas veces los aprendizajes más importantes los llevan en su interior, es decir, no por más hacer saben más" (C14).

- "Procura hacer interesante su enseñanza, despierta el deseo de trabajar" (C 16).

- "Persona justa para calificar tareas y exámenes Exige que el trabajo esté bien hecho en el tiempo debido. Señala tareas razonables y luego las revisa." (C 16).

- "Es franco y recto en el trato con sus alumnos." (C16).

Otro de los elementos que configuran la imagen del profesor es el prestigio social percibido. Al respecto, muy pocos participantes consideran que es una profesión bien valorada: "tiene una imagen social positiva" (C08); "...está bien valorado, ya que muchos padres agradecen nuestra labor" (CO1) "en términos generales, buena..." (C05). 
La mayoría afirma que la imagen social es negativa: "mala, socialmente está muy poco reconocido nuestro trabajo" (C04); "poco valorado" (C09; C15); "en general, es mala" (C13). Muchos consideran que ha habido un empeoramiento en el reconocimiento social del maestro (C09; C10; C11; C14), llegando, incluso, a no ser respetado como profesional:

- "Pienso que se ha perdido respeto a la figura del profesor y que muchas veces no se tiene en cuenta lo que decimos. Además creo que se nos ridiculiza y se nos falta al respeto delante de los alumnos." (C03).

- "De cuando yo era estudiante de la EGB a ahora, han pasado muchos años. Antes teníamos respeto a los profesores y éstos además tenían el apoyo de nuestros padres, hoy en día es todo lo contrario. El alumno, no tiene respeto por el profesor y los padres se ponen a favor de su hijo, únicamente escuchando la versión de éste y no la de la escuela. (C06).

- "... comparado con la imagen que tenía en épocas anteriores el papel del profesor ha sido degradado hasta puntos insospechables, hoy en día se tiene la imagen de que eso lo hace cualquiera" (CO7).

- 'La sociedad ha perdido la 'admiración' que se debe tener a la persona capaz de traspasar conocimiento y de estimular al aprendizaje. Somos lo que tenemos en nuestra cabeza y ello depende en gran medida de lo que te enseñan y aprendes" (C15).

Esta falta de prestigio social se concreta, según opinión de los participantes, en una consideración generalizada de que no trabajan lo suficiente:

- "...hay gente que sigue pensando que trabajamos poco y tenemos muchas vacaciones" (C01).

- "Que vivimos muy bien. Que no trabajamos mucho. Que cobramos muy bien. Que tenemos muchas vacaciones. En definitiva, que es un chollo" (CO2).

- "Lo único en lo que piensan es en el dinero y las vacaciones." (C07). 
La imagen profesional del profesorado en formación continua.

- "Que tienen muchas vacaciones y que viven muy bien. En general no hacen gran cosa" (C12; C14).

- "... que vivimos como reyes, tenemos muchas vacaciones, puentes, dias festivos... Algunos piensan que somos unos vagos, que nuestro trabajo no requiere ningún esfuerzo y que nos quejamos de vicio." (C16).

- Alguno menciona que no se valora adecuadamente su formación: "estos en la uni aprenden a pintar, a cantar! Qué fácil magisterio!" (C05).

Las respuestas indican que no se sienten apoyados por los padres, a pesar de que muchas veces tienen que asumir tareas que correspondería a la familia:

- "Hoy en día los padres tienen la tendencia a sacar la cara a sus hijos poniendo en entredicho nuestra forma de actuar y nuestra metodología de enseñanza aprendizaje." (C03).

- "En muchos casos son los encargados de reeducar a los niños y esa tarea les impide poder desempeñar su actividad como debieran." (C11).

En resumen, cuando se pide a los participantes que definan la profesión, unos lo hacen desde la satisfacción que les produce, otros inciden en la importancia de la misma, y algunos especifican las funciones, insistiendo en la función de guía.

El análisis de las metáforas indica que algunos consideran al maestro como fuente de conocimiento, otros como un guía, otros como un recurso; algunos reflejan la dedicación que implica y otros su descontento.

Las características del buen profesor se pueden condensar en el dominio de ciertas habilidades sociales, en el interés por mejorar e innovar continuamente, en una actitud de preocupación por el alumno y en su cercanía, en ciertas características personales como la paciencia y la amabilidad, y en determinadas formas de ejercer sus tareas, motivando y dejando autonomía al estudiante.

Con respecto al prestigio social percibido, la mayoría de los participantes considera que es una profesión poco valorada. 


\subsection{Evolución de la imagen del profesor a partir de la experiencia}

Algunos participantes declaran que su imagen "es prácticamente la misma" (C04; C05; C08), "intentando que nada ni nadie desmorone la imagen del docente que tenían y tienen" (C05).

Algunos encuestados valoran más la profesión (C08) porque el desempeño profesional se ha vuelto más complejo y exigente:

- "Antes de empezar creía que era algo más sencillo; ahora me doy cuenta que cuanto más me involucro en este ámbito más me doy cuenta de lo complicado que es (C13).

- "Se ha apreciado un cambio importante, tanto en el tipo de alumnado, el número de inmigrantes, el trato del alumno y sus familiares hacia el profesor" (C07; "...el tratar con algunos padres cada vez se me hace más difícil" (C04).

- "... cada día se pide que se sepa más pero luego tenemos muy poco tiempo para poder estar con los chavales y solucionar los problemas" (C 12).

- "Antes el profesor era una figura más lejana y hoy en día se ve más involucrado tanto dentro como fuera del colegio en la educación de sus alumnos" (C09).

La experiencia variada ha hecho a algunos maestros y maestras enriquecer su perspectiva profesional: "siendo sustituta tienes la oportunidad de conocer diferentes colegios y diferentes profesores, escogiendo lo mejor de cada uno de ellos e integrándolo dentro de tu quehacer diario" (C10).

Asimismo, la experiencia les ha permitido ser realistas en la concepción de la profesión:

- "... he podido observar que hay toda clase de profesores, de los que aún se implican con sus alumnos y de los que dan la clase sin observar la aptitud de sus alumnos. Pero son más los que se preocupan por sacar adelante a sus alumnos" (C06).

- "...he apreciado un cambio importante en la desidia del personal que lleva muchos años y ha estado sujeto a todos estos cambios de los diferentes sistemas educativos" (C07).

- "...con la edad y la experiencia el talante va cambiando" (C16). 
La imagen profesional del profesorado en formación continua.

El recorrido profesional y las exigencias actuales conducen a algunos maestros y maestras a apostar por la mejora continua:

- "Hay que apostar por la innovación y mejora continua para poder responder a los intereses de los alumnos de hoy en día y de la sociedad" (CO3).

- "Creo que no nos tenemos que estancar, tenemos que intercambiar experiencias, reciclarnos... porque somos responsables de un grupo de niñas y niños a los que les espera un futuro en el que de una manera $u$ otra manera podemos influir de manera positiva o negativa" (C14).

En resumen, la experiencia docente permite construir una imagen más realista y completa de la profesión, lo que, en ocasiones, favorece la consciencia de la necesidad de formarse y de innovar y mejorar continuamente.

\section{CONCLUSIONES Y DISCUSIÓN}

Los profesores participantes enumeran muchas características que debe tener un profesor. Haciendo un análisis del conjunto se pueden agrupar siguiendo la diferenciación que hace Echeverría (2005) de la competencia de acción profesional. En la competencia técnica y metodológica radica el "SABER" del profesional. De las elementos competenciales señalados por los participantes se incluyen en este grupo los conocimientos pedagógicos y disciplinares.

Cuando a los profesores se les ha preguntado sobre lo que un docente tiene que saber hacer, la competencia metodológica, en términos de Echeverría (2005), han respondido con "saberes" que no sólo se refieren al saber hacer (escuchar, motivar, conocer al otro, interesar, respetar ritmos, ser comprensivo) del docente sino también a la competencia técnica (tener conocimientos, transmitir).

Hay otras características del profesor que componen según Echeverría (2005: 3) "ese especial SABOR que caracteriza a los mejores profesionales". Entre ellas, están: amabilidad, pasión, cercanía, empatía, comprensión, respeto al otro, paciencia, cariño, dinamismo, motivación, flexibilidad, tranquilidad, actividad, ilusión, dedicación, alegría y optimismo, fundamentales e imprescindibles para el profesional de la educación, para un buen profesor. 
Los profesores participantes afirman que el docente tiene que ser competente técnica y metodológicamente ("mejora continua", "ser capaz"). Pero fundamentalmente tiene que ser y actuar de una forma concreta ("amabilidad, pasión, cercanía, empatía, comprensión, respeto al otro, paciencia, cariño, dinamismo, motivación, flexibilidad, tranquilidad, actividad, ilusión, dedicación, alegría y optimismo"). Es decir, tiene que "saber" y tener "sabor" (Echeverría, 2005).

Los profesores consideran que su profesión es mucho más que enseñar contenidos. Algunos ejemplos que apoyan esta afirmación son las metáforas que eligen para definir al profesor, cuyo significado está vinculado al apoyo y la orientación, entre otras.

Estas características coinciden en gran parte con el perfil del profesor mediador descrito por Tébar (2003) que se basa en generar motivación e implica a los estudiantes en su propio proceso educativo. Este autor señala las cualidades indispensables en el enseñante: competencia pedagógica, madurez y estabilidad emocional, conocimiento de la materia que va enseñar, comprensión de los procesos del desarrollo del niño, preocupación y respeto hacia las personas de los alumnos, capacidad de adaptación al equipo docente, toma de consciencia de escuela, situada en su marco social, y espíritu abierto y dinámico.

Los profesores señalan dificultades relacionadas con los recursos como el tiempo, la falta de personal o el número elevado de estudiantes, así como dificultades relacionadas con la falta de motivación de los estudiantes y las actitudes y comportamientos de las familias. En opinión de Marchesi (2012:13) "no es fácil que un profesor competente y motivado pueda mantener una experiencia profesional satisfactoria si el resto de las condiciones no acompaña a su esfuerzo". Para superar estas dificultades el docente necesita tener vocación (Marques, 2006), pasión (Day, 2006) y recursos personales y materiales.

Por el contrario, cuando a los profesores se les ha preguntado por los aspectos que les ayudan en el desarrollo de sus funciones han aludido al clima, el apoyo de los compañeros y la pasión por la educación y por la materia que enseña, resultado confirmado por Mañu y Guyarrola (2011).

Los profesores tienen que poseer un conjunto de competencias para desempeñar sus funciones. Dichas competencias se empiezan a desarrollar en la formación inicial pero esta adquisición es "un proceso de construcción a lo largo de su recorrido profesional" (Tardif, 2004:12). Como afirman Yanes y Ríes (2013) la experimentación práctica es 
La imagen profesional del profesorado en formación continua.

fundamental en la formación inicial para que el futuro profesor se sienta más competente y mantenga la necesidad de la formación continua para mejorar el desempeño de su profesión.

Aun considerando las limitaciones de este estudio, como el número de participantes y las peculiaridades del procedimiento de captación de la muestra, los resultados obtenidos en esta investigación pueden orientar en la construcción de una oferta formativa para el profesorado más acorde a su pensamiento sobre la profesión docente. La identidad profesional del docente debe ser una temática a trabajar en las actividades formativas dirigidas al profesorado en activo de tal manera que la formación se convierta en "una movilización de experiencias adquiridas en su carrera, cuya reutilización con significados distintos genere nuevos saberes" (Bolívar, 2006:37).

\section{REFERENCIAS BIBLIOGRAFICAS}

Beijaard, D., Meijer, P. C. y Verloop, N. (2004). Reconsidering research on teachers'professional identity. Teaching and Teacher Education, 20, 107-128. D.O.I.: http://dx.doi.org/10.1016/j.tate.2003.07.001

Bolívar, A. (2006). La identidad profesional del profesorado de secundaria, crisis y reconstrucción. Málaga: Aljibe.

Day, C. (2005). Formar docentes: cómo, cuándo y en qué condiciones aprende el profesor. Madrid: Narcea.

Day, C. (2006). Pasión por enseñar: la identidad personal y profesional del docente. Madrid: Narcea.

Day, C. y Gu, Q. (2012). Profesores: vidas nuevas, verdades antiguas. Una influencia decisiva en la vida de los alumnos. Madrid: Narcea.

De la Calle, M.J. (2004). El reto de ser profesor en el contexto de la convergencia europea. Revista Interuniversitaria de Formación del profesorado, 18, (3), 251-258.

Echeverría, B. (2002).Gestión de la competencia de acción profesional. Revista de Investigación educativa, 20, (1), 7-43.

Echeverría, B. (2005). La competencia de acción de los profesionales de la orientación. Madrid: ESIC.

Galaz, A. (2011). El profesor y su identidad profesional ifacilitadores $U$ obstáculos del cambio educativo? Estudios Pedagógicos XXXVII, 2, 89-107.

Harris, L. (2011). Secondary teachers' conceptions of student engagement: Engagement in learning or in schooling? Teaching and Teacher Education, 27, (2), 376-386.

D.O.l.: http://dx.doi.org/10.1016/i.tate.2010.09.006 
Lauriala, A., y Kukkonen, M. (2005). Teacher and student identities as situated cognitions. En P. Denicolo y M. Kompf (Eds.), Connecting policy and practice: Challenges for teaching and learning in schools and universities (199-208). Oxford: Routledge. Citado por Beaucham, C. y Thomas, L. (2009). Understanding teacher identity: an overview of issues in the literature and implications for teacher education. Cambridge Journal of Education, 39, (2), 175189.

Mañu, J.M. y Goyarrola, I. (2011). Docentes competentes: por una educación de calidad. Madrid: Narcea.

Marcelo, C. y Vaillant, D. (2011). Desarrollo profesional docente: ¿̇cómo se aprende a enseñar? Madrid: Narcea.

Marchesi, A. (2012). El bienestar de los docentes en tiempos de crisis. Revista Fuentes (Sevilla), 12, 9-12.

Marques, R. (2006). Un arte y una vocación. Madrid: Narcea.

Palmer, P.J. (1998). The Courage to Teach: Exploring the Inner Landscape of a Teacher's Life. San Francisco: Jossey-Bass.

Sáez, J. y García, J. (2011). Metáforas del educador. Valencia: Nau Llibres.

Sachs, J. (2005). Teacher education and the development of professinal identity: Learning to be a teacher. En P. Denicolo y M. Kompf (Eds.), Connecting policy and practice: Challenges for teaching and learning in schools and universities (5-21). Oxford: Routledge.

Sutherland, L. y Markauskaite, L. (2012). Examining the role of authencity in supporting the development of professional identity: an example from teacher education. Higher Education, 64, 747-766.

D.O.I.: http://dx.doi.org/10.1007/s 10734-012-9522-7

Tardif, M. (2004). Los saberes del docente y su desarrollo profesional. Madrid: Narcea.

Tébar, L. (2003). El perfil del profesor mediador. Madrid: Santillana.

Tiana, A. (2011). La Formación de Postgrado del profesorado de Enseñanza Secundaria. Revista Fuentes (Sevilla), 11, 13-27.

Timostsuk, I., Ugaste, A. (2012). The role of emotions in student teachers professional identity. European Journal of Teacher Education, 35, (4), 421-433.

D.O.I.: http://dx.doi.org/10.1080/02619768.2012.662637

Walkington, J. (2005). Becoming a teacher: encouraging development of teacher identity through reflective practice. Asia-Pacific Journal of Teacher Education, 33, (1), 53-64.

D.O.I.: $\mathrm{http} / / / \mathrm{dx} . \mathrm{doi} . \mathrm{org} / 10.1080 / 1359866052000341124$

Yanes, C. y Ries, F. (2013). Liderando el cambio: estudio sobre las necesidades formativas de los futuros docentes de secundaria. Revista Fuentes (Sevilla), 14, 105-124. 


\section{Sobre los autoras:}

\section{Zoe Martínez de la Hidalga}

zoe@deusto.es

Licenciada en Pedagogía. Profesora del Departamento de Didáctica y Desarrollo Curricular de la Facultad de Psicología y Educación de la Universidad de Deusto, Investigadora del Equipo Desarrollo de Competencias y Valores. Profesora y Directora del Máster en Formación del Profesorado de Educación Secundaria. Es responsable de la Formación Continua del Profesorado No Universitario en la Facultad de Psicología y Educación de la Universidad de Deusto, siendo una de sus líneas de investigación la identidad profesional y el desarrollo del profesorado.

\section{Lourdes Villardón-Gallego}

lourdes.villardon@deusto.es

Catedrática del Departamento de Didáctica y Desarrollo Curricular de la Facultad de Psicología y Educación de la Universidad de Deusto e Investigadora Principal del Equipo Desarrollo de Competencias y Valores. Es profesora del Máster de Profesorado de Educación Secundaria. Ha desarrollado múltiples actividades de formación continua de profesorado y asesoramiento a centros docentes, fundamentalmente en temáticas relacionadas con Metodologías Didácticas Activas y Desarrollo y Evaluación de Competencias.

\section{Para citar este artículo:}

Martínez, Z. y Villardón-Gallego, L. (2014). La imagen profesional del profesorado en formación continua. Revista Fuentes, 15, Junio, pp. 187212. [Fecha de consulta: $\mathrm{dd} / \mathrm{mm} / \mathrm{aaaa}$ ]. http://www.revistafuentes.es/ D.O.I.: http://dx.doi.org/10.12795/revistafuentes.2014.i15.09 